\title{
Marché libre, rendement collectif et intérêts privés, un trio infernal ? Les fermiers des assises de la ville de Gand à la fin du Moyen Âge ${ }^{1}$
}

\author{
Marc Boone
}

\section{La fiscalité urbaine : l'écrasante prépondérance des impôts indirects, les assises}

Les impôts indirects ou assises représentaient à eux seuls l'écrasante majorité des recettes de la plupart des villes médiévales : des proportions de 75 à $80 \%$ des revenus annuels n'étaient pas exceptionnels ${ }^{2}$. Les villes des anciens Pays-Bas (et du comté de Flandre) ne faisaient pas exception ${ }^{3}$. Cette pratique ne s'opposait pas à la vocation marchande des villes, bien au contraire : elle permettait de diviser le poids de la fiscalité entre tous les consommateurs, riches et pauvres, et en plus elle offrait aux élites marchandes, qui avaient généralement une influence certaine sur les décisions politiques, la possibilité de protéger leurs propres produits et de taxer ceux des concurrents de l'extérieur ${ }^{4}$. La percée d'une économie supposée plus moderne dite de capitalisme commercial, car axée sur le

1 Article conçu dans le cadre des programmes PAI (Pôles d'attraction interuniversitaire) financés par la politique scientifique fédérale belge, que j'ai l'honneur de diriger, voir : www.cityandsociety.be pour la dernière phase VII du programme. Le texte est une mise au point d'un texte paru en néerlandais il y un quart de siècle : M. BOone, « Triomferend privéinitiatief versus haperend overheidsoptreden? Over pachters van indirecte belastingen in laatmiddeleeuwse steden ", Tijdschrift voor sociale geschiedenis, 15, 1989, p. 113-138. Une première version en français en a été présentée lors d'une table ronde « Fiscalidad urbana y elites financieras en el Occidente medieval (siglos XIV-Xv)» tenue à Barcelone, les 2829 octobre 2010 à l'initiative d'un ami commun, Manuel Sánchez Martínez (CSIC, Institut Mila y Fontanals).

2 Voir en général: F. Irsigler, «Aksize », Lexikon des Mittelalters, 1, 1980, col. 26. Le constat a été confirmé depuis lors dans presque chaque étude consacrée aux finances d'une ville médiévale.

${ }^{3}$ Sur ces villes en général (avec une attention particulière pour les finances urbaines) : M. Boone, À la recherche d'une modernité civique. La société urbaine des anciens PaysBas au bas Moyen Âge, Bruxelles, Éditions de l'ULB, 2010, p. 124-135. 
commerce international, incarnée successivement par des villes comme Anvers et Amsterdam dans le courant $\mathrm{du} \mathrm{xvI}^{\mathrm{e}}$ siècle, n'a en rien altéré la position dominante des taxations indirectes à l'intérieur des deux métropoles commerciales. Dans les décennies 1530-1545, la "grande » assise, levée de préférence sur la consommation de la bière, représentait $80,6 \%$ des revenus à Anvers, 71,8\% à Amsterdam; les assises dites « menues » représentant en plus, respectivement dans les deux villes, 8,3 et $17 \%$ des revenus 5 .

Les assises étaient impopulaires et dès la fin du xiII ${ }^{e}$ siècle les témoignages concernant la condamnation de cette forme d'imposition se retrouvent fréquemment ${ }^{6}$. Dans la plupart des révoltes urbaines, qui, en ce qui concerne l'ancien comté de Flandre, vont crescendo jusqu'au paroxysme de 1302, des échos d'une conscience d'être sujet à un prélèvement qui pénalise la consommation courante et ne touche que beaucoup trop peu les grandes fortunes sont abondants. Premier paradoxe qu'il faut souligner : l'arrivée au pouvoir (ou plutôt la participation à l'exercice du pouvoir) des corps de métier en Flandre, qui fut le résultat le plus tangible de cette première grande vague de rébellions urbaines, ne se traduisit pas par l'abolition des assises comme formule fiscale de prédilection. Traditionnellement, les corps de métier comptaient dans leurs rangs les représentants les plus critiques et les plus opposés à la gestion financière du patriciat urbain traditionnel, dont les assises formaient la clé de voûte ${ }^{7}$. Qu'ils n'aient pas profité de leur participation au pouvoir pour abolir un système passablement critiqué, pour ne pas dire haï, peut surprendre. Ce constat force à poser quelques questions : d'abord, était-on en mesure d'opérer un changement profond (vers une fiscalité directe par exemple), et si non, a-t-on, même si on n'a pas changé le système, profité de l'occasion pour en modifier quelques modalités?

\footnotetext{
J.-Cl. Hocquet, « Cité-État et économie marchande », dans R. Bonney (dir.), Systèmes économiques et finances publiques, Paris, Presses Universitaires de France/European Science Foundation, 1996 (les origines de l'État moderne en Europe, $\mathrm{XII}^{\mathrm{e}}-\mathrm{XVIII}{ }^{\mathrm{e}}$ siècle), p. 71.
}

5 Voir M. C. 'T Hart, M. Limberger, «Staatsmacht en stedelijke autonomie. Het geld van Antwerpen en Amsterdam (1500-1700)», Tijdschrift voor sociale en economische geschiedenis, 3, 2006, p. 43.

${ }^{6}$ Une dernière mise au point par C. BILlen, «À la recherche d'un prélèvement fiscal équitable. Pratiques, discours et porte-parole, dans les Pays-Bas méridionaux ( $\mathrm{XIII}^{\mathrm{e}}$ $\mathrm{XIV}^{\mathrm{e}}$ siècles) », dans S. CAVACIOCCHI (dir.), La fiscalità nell'economia Europea secc. XIII-XVIII. Fiscal Systems in the European Economy from the 13th to the 18th Century, Firenze, 2008 (Fondazione Istituto internazionale di storia economica F. Datini, Prato, Serie II Atti delle 'settimane di studi' 39), p. 871-880. 


\section{L'improbable alternative des impôts directs}

Donner la préférence à une fiscalité directe, fondée sur un prélèvement en fonction des fortunes (immobilières, en rentes ou en revenus) dépassait largement les possibilités réduites des chancelleries urbaines en place dans les anciens Pays-Bas. Même dans des grandes villes à l'échelle européenne comme les villes flamandes de Bruges et Gand, le nombre de technocrates actifs au sein des administrations urbaines demeurait très restreint ${ }^{8}$. La recherche concernant les chancelleries urbaines étant encore à ses débuts, ce qu'on sait déjà concernant le nombre et les capacités des comptables et autres employés formés aux techniques fiscales, révèle des moyens administratifs modestes et une impossibilité d'organiser à court terme une réorientation profonde. Les rares épisodes, souvent de crise aiguë, pendant lesquels on a expérimenté une taxation

\footnotetext{
7 Voir une synthèse concernant cette évolution dans le contexte du conflit Franco-flamand du début du XIV ${ }^{\mathrm{e}}$ siècle : M. Boone, «Une société urbanisée sous tension. Le comté de Flandre vers 1302 », dans R.C.V VAN CAENEGEM (dir.), 1302. Le désastre de Courtrai. Mythe et réalité de la bataille des Eperons d'or, Anvers, Fonds Mercator, 2002, p. 52-53 (sur la fiscalité urbaine). Le contexte des révoltes urbaines (avec une connotation fiscale indéniable) : M. Boone, «Le comté de Flandre dans le long $\mathrm{XIV}^{\mathrm{e}}$ siècle : une société urbanisée face aux crises du bas Moyen Âge », dans M. Bourin, G. Cherubini et G. Pinto (éd.), Rivolte urbane e rivolte contadine nell'Europa del Trecento: un confronto, Firenze, Firenze University Press, 2008, p. 17-47. Une approche privilégiant l'analyse discursive a également mis l'accent sur l'aspect fiscal de la contestation : J. Dumolyn, J. Haemers, « A Bad Chicken Was Brooding. Subversive Speech in Late Medieval Flanders », Past \& Present, 214, 2012, p. $70-77$.
}

${ }^{8}$ La diplomatique urbaine a été longtemps un parent pauvre de la diplomatique comme science auxiliaire. Un colloque tenu à Gand il y quelques années a cherché à remédier à cette situation: W. Prevenier et Th. de Hemptinne (éd.), La diplomatique urbaine en Europe au moyen âge. Actes du congrès de la Commission Internationale de Diplomatique, Gand, 25-29 août 1998, Leuven, 2000, XIV-581 p. On y ajoutera une thèse récente concernant la ville brabançonne de Bois-le-Duc, de G. VAN Synghel, Actum in camera scriptorum oppidi de Buscoducis. De stedelijke secretarie van 's-Hertogenbosch tot ca. 1450, Hilversum, Verloren, 2007, passim. Des recherches en cours (Tineke Van Gassen pour Gand et Valeria Van Camp pour Mons notamment) sont en train d'y remédier. Voir par exemple l'édition et étude de L. Gaudreault, Pouvoir, mémoire et identité. Le premier registre de délibérations communales de Brignoles (1387-1391), édition et analyse, Montpellier, PULM, 2014; l'article de fond de M. H. D. C. Coelho, «Writing in the Urban World», História (Saõ Paulo), 34, 1, jan./jun. 2015, p. 16-34, http://dx.doi.org/10.1590/1980-43692015 0001000038 , ainsi que V. VAN CAMP, «La diplomatique des comptes : méthode, limites et possibilités. L'exemple de Mons, $\mathrm{XIV}^{\mathrm{e}}-\mathrm{XV}^{\mathrm{e}}$ siècles ", Archiv für Diplomatik, 61, 2015, p. 237 270, et T. VAN GASSEN, "City Cartularies in Late Medieval Ghent: A Sign of Urban Identity? », dans E. C. Dıјног (éd.), Medieval Documents as Artefacts. An Interdisciplinary Perspective on Codicology, Palaeography and Diplomatics, Hilversum, Verloren, à paraître. 
directe dans les villes, n'ont pas été très convaincants en ce qui concerne un éventuel remplacement des impôts indirects. Une fiscalité directe performante aurait en effet supposé un recensement des patrimoines et l'identification des contribuables. Or, les rares occasions qui permettent de mesurer l'impact probable d'une telle mise en œuvre, souvent en relation avec une conjoncture bien spécifique (situation de guerre ou de crise extrême), nous apprennent qu'en tout état de cause, les grandes villes des Pays-Bas perdaient presque autant qu'elles gagnaient en montant une telle opération.

Les années de crises politico-militaires qui ont suivi l'annonce de la mort inopinée du duc de Bourgogne Charles le Téméraire en 1477 et la guerre de succession qu'a dû livrer son gendre Maximilien d'Autriche afin de sauvegarder son héritage aux Pays-Bas nous livrent des exemples de telles expérimentations. Pour les deux grandes villes de Flandre, la ville marchande de Bruges et la ville plus industrielle de Gand, nous disposons d'une analyse récente qui montre que dans les deux cas les villes se sont vues obligées de recourir à une taxation directe, à côté bien sûr des impôts indirects classiques. Différentes stratégies s'offraient alors : ou bien la ville suivait la piste de l'obligation (on procédait alors à des emprunts forcés, misant sur un réflexe presque républicain, auprès des nantis qui s'engageaient sans espoir de recevoir des intérêts sur les sommes mises à disposition de la communauté), ou bien on s'engageait dans la voie du volontarisme fiscal en vendant des rentes ou en contractant des emprunts spontanés contre intérêt ${ }^{9}$. Dans le cas de Bruges, on a choisi la voie du volontarisme, parce qu'aucune tradition administrative n'existait qui aurait permis de s'engager sur la voie d'une véritable fiscalité directe. Étonnant à première vue, étant donné la volonté politique affichée en 1477 de s'engager dans cette direction, vu l'emprise retrouvée des représentants des corps de métier sur la politique et la gestion financière de la ville, après en

\footnotetext{
${ }^{9}$ Les deux possibilités (la ville république ou la ville-État, comme il les présente) ont été retenues aussi par J. TRACY, " On the Dual Origins of Long-Term Urban Debt in Medieval Europe ", dans M. Boone, K. Davids et P. Janssens (éd.), Urban Public Debts. Urban Government and the Market for Annuities in Western Europe (1 $4^{\text {th }}-18^{\text {th }}$ Centuries), Turnhout, Brepols, 2003 (Studies in European Urban History, 1100-1800, 3), p. 24. Pour Bruges, on trouvera une analyse détaillée dans la thèse de J. Haemers, For the Common Good. State Power and Urban Revolts in the Reign of Mary of Burgundy (1477-1482), Turnhout, Brepols, 2009 (Studies in European Urban History, 1100-1800, 17), p. 195-203. En ce qui concerne les rentes vendues par la ville de Bruges, voir L. Derycke, « The Public Annuity Market in Bruges at the End of the $15^{\text {th }}$ Century », dans Urban Public Debts..., op. cit., p. 165-181.
} 
avoir été écartés pendant des décennies ${ }^{10}$. N'empêche que la majorité écrasante des deniers qui entraient dans les caisses brugeoises provenait de la fiscalité indirecte classique, les "assises » levées sur les produits de consommation on ne peut plus traditionnels : bière, blés, tourbe, vin etc ${ }^{11}$.

La crise de l'État bourguignon et le privilège général, ainsi que les privilèges pour chaque principauté et les privilèges spécifiques pour quelques grandes villes (comme Bruges et Gand) obtenus de la duchesse Marie au début de l'année 1477 par les sujets, ont marqué les esprits des contemporains et avant tout ceux des élites artisanales qui en furent les forces inspiratrices. Ils autorisaient le retour à la situation d'avant le principat autocratique du feu duc Charles le Téméraire ${ }^{12}$. N'empêche que dans le cas de Bruges, les lendemains ne chantèrent pas : cinq mois plus tard seulement, le gouvernement de la ville réinstallait le régime fiscal en vigueur sous le Téméraire. Dès le $1^{\text {er }}$ mai, les assises sur le vin avaient été rehaussées. Dès le $1^{\text {er }}$ août 1477 , les assises socialement explosives sur la bière et sur le grain (produits de consommation populaire par excellence) atteignirent le niveau d'avant «1477» pour le dépasser de façon spectaculaire dans les années suivantes ${ }^{13}$. C'est que, sous la pression des nécessités avant tout militaires, même les représentants des corps de métier - les plus farouches opposants au régime fiscal mis en place sous le Téméraire - s'étaient convertis en défenseurs d'une politique de résistance

\footnotetext{
${ }^{10}$ Notons que les représentants des métiers eux-mêmes doutaient clairement de l'efficacité du procédé. Après avoir formulé le principe d'un prélèvement de denier $100^{\text {ième }}$ sur les avoirs et fortunes des Brugeois, ils avaient accordé un délai d'un mois afin de pouvoir étudier les effets escomptés : J. Haemers, For the Common Good..., op. cit., p. 196-197.

${ }^{11}$ Voir J. Haemers, For the Common Good..., op. cit., p. 196. Pendant la période 1476-1482 en moyenne $57,06 \%$ des revenus de la ville étaient fournis par les assises, avec des pics allant jusqu'à $70 \%$. Cette moyenne relativement basse est explicable par les prélèvements exceptionnels, le résultat des crises militaires et autres de ces années.

${ }^{12}$ De ces textes émane une volonté très prononcée de modifier l'emprise fiscale de l'État sur les économies urbaines : voir les éditions et un commentaire de fond élaborant une lecture «constitutionnelle» des textes par W. Blockmans, 1477. Le privilège général et les privilèges régionaux de Marie de Bourgogne pour les Pays-Bas, Courtrai ,1985 (Anciens pays et assemblées d'États, LXXX), passim. Concernant la relation entre le Téméraire et les villes : M. Boone, "Charles le Téméraire face au monde urbain : ennemis jurés et fatals ? ", dans K. Oschema, R. C. Schwinges (dir.), Karl der Kühne von Burgund. Fürst zwischen europäischem Adel und der Eidgenossenschaft, Zürich, NZZ libro, 2009, p. 185-201.
}

${ }^{13}$ Voir les chiffres éloquents chez J. HaEmers, For the Common Good..., op. cit. p. 202. 
armée et de rigueur budgétaire. Seul le maintien d'une pratique de consultation large a pu adoucir les effets socio-politiques : un an après le retour au régime fiscal du Téméraire, en août 1478, une commission élargie comptant 150 membres, issus aussi bien des rangs des gens de métier que de la "bourgeoisie» au sens socialement plus limité, avait donné le feu vert à une augmentation sensible des assises existantes (sur la bière et les blés) et à l'instauration d'une assise nouvelle (un dixième à lever sur la vente de la viande et du poisson). Même image globale à Gand : là aussi la crise de l'État bourguignon et la transition difficile vers la dynastie des Habsbourg a provoqué un recours exceptionnel à la fiscalité directe.

En plusieurs vagues, Gand, qui restait la plus grande ville des anciens Pays-Bas avec une population qui tournait autour de 50000 habitants, s'est vue obligée de recourir à une fiscalité directe, mélangeant plusieurs formules: un prélèvement uniforme et forfaitaire sur chaque famille ou feu, un emprunt forcé et finalement un prélèvement proportionnel à la richesse en biens immobiliers. Chaque formule restait toutefois soumise à une restriction de taille, à savoir que les capacités administratives ne permettaient pas de globaliser le patrimoine d'un propriétaire au-delà de la circonscription administrative, une paroisse, ou une « connétablie », division territoriale et militaire de la ville ${ }^{14}$. Il en résulte un rendement faible, mais cependant réel: les sommes ainsi collectées servaient à répondre aux besoins militaires souvent très urgents et évitaient le recours encore plus massif aux ventes de rentes ou à une augmentation sensible des assises, toujours très délicate dans une ville à la réputation bien méritée de rebelle. Ce qui n'empêche pas, aussi dans le cas de Gand, que les assises soient restées la colonne vertébrale des finances urbaines.

\footnotetext{
${ }^{14}$ La dernière synthèse nous est offerte par W. Rycквоsch, Tussen Gavere en Cadzand. De Gentse stadsfinanciën op het einde van de middeleeuwen (1460-1495), Gand, 2007 (Verhandelingen van de Maatschappij voor Geschiedenis en Oudheidkunde te Gent, XXXI), p. 100-117. La dernière vague de taxations directes a fait l'objet d'une analyse sociale : $\mathrm{M}$. Boone, M. Dumon, B. Reusens, Immobiliënmarkt, fiscaliteit en sociale ongelijkheid te Gent, 1483-1503, Courtrai, 1981 (Anciens pays et assemblées d'États, LXXVIII), 285 p. Certains documents on fait l'objet d'une édition: M. Boone, "De Gentse verplichte lening van 1492-1493 ", Bulletin de la Commission Royale d'Histoire, CXLVII, 1981, p. 247-305 et $\mathrm{W}$. Blockmans, Peilingen naar de sociale structuren te Gent tijdens de late $15^{e} e e u w$, Courtrai, 1971 (Anciens pays et assemblées d'États, LIV), W. Blockmans, De vermogensstructuur in de Sint-Jakobsparochie te Gent, 1492-1494, Courtrai, 1973 (Anciens pays et assemblées d'États, LXIII), passim.
} 
Une fois encore au milieu $\mathrm{du} \mathrm{xvI}^{\mathrm{e}}$ siècle, le gouvernement de Philippe II et de ses représentants aux Pays-Bas a bel et bien essayé de modifier profondément le système fiscal en vigueur dans les villes. Ces tentatives de la part du gouvernement central d'introduire de nouvelles taxations et de rationaliser les systèmes existants rencontraient toujours plus de résistance. Même si, toutes proportions gardées, elles auraient signifié à la fois une rationalisation et une répartition plus équitable des charges, comme ce fut le cas pour le projet du duc d'Albe d'introduire, en 1569 , un centième denier sur les revenus ${ }^{15}$. Mais comme l'avait déjà remarqué Jan Craeybeckx, «après la pacification de Gand de 1576, Guillaume d'Orange et ses partisans arrachèrent sans grandes difficultés les impôts que Charles Quint et Philippe II n'avaient que rarement obtenus des États $^{16}$ ». Ces derniers ont même accordé la levée des «moyens généraux », des taxes uniformes dans toutes les provinces. Les sujets, par le truchement des décisions des élites censées les représenter, étaient donc disposés à payer les impôts à l'État, mais pas à n'importe qui, ni dans n'importe quel contexte. Fondamental était le fait qu'ils pouvaient continuer à décider du mode d'imposition à utiliser à l'intérieur des villes pour finalement réaliser le prélèvement souhaité. Or ce mode était et restait la taxation indirecte, moyennant des assises sur la consommation et les transactions

\section{Les impôts indirects : entre gain privé et intérêt public, un vrai ou un faux débat?}

Le prélèvement d'assises pouvait être organisé sous deux formes. La ville pouvait s'appuyer sur des officiers assermentés pour collecter les revenus en question, ce qui semble avoir été le cas dans les villes du sud de

\footnotetext{
${ }^{15}$ Sur cette tentative et les difficultés qu'elle a rencontrées, on consultera toujours avec grand profit l'article classique de J. CraeYbeckx, «La portée fiscale et politique du $100^{\mathrm{e}}$ denier du duc d'Albe ", Acta Historica Bruxellensia. Recherches sur l'histoire des finances publiques en Belgique, I, 1967, pp. 343-374. L'entreprise du duc d'Albe s'inscrit dans une série de tentatives de réformes tout au long du $\mathrm{XvI}^{\mathrm{e}}$ siècle, voir W. Blockmans, «Finances publiques et inégalité sociale dans les Pays-Bas aux $\mathrm{xIV}^{\mathrm{e}}-\mathrm{XVI}^{\mathrm{e}}$ siècles ", dans J.-Рн. Genet et M. Le Mené, (éd.), Genèse de l'État moderne. Prélèvement et redistribution. Actes du colloque de Fontevraud 1984, Paris, CNRS, 1987, p. 81-82. Voir aussi l'édition des registres du $100^{\mathrm{e}}$ denier: P. Stabel, F. Vermeylen, Het fiscale vermogen in Brabant, Vlaanderen en in de heerlijkheid Mechelen : de honderdste penning van de hertog van Alva (1569-1572), Bruxelles, 1997 (CRH, in-8º).
}

${ }^{16}$ J. Craeybeckx, « La portée fiscale et politique... », art. cit., p. 363. 
l'Empire, dans le Haut Rhin et dans la Confédération helvétique notamment ${ }^{17}$. Dans les villes des anciens Pays-Bas, par contre, la collecte par des officiers était l'exception, car les villes avaient une préférence massive et très généralisée pour la formule qui consistait à donner les assises à ferme aux plus offrants ou en réalité à un consortium de «fermiers» qui étalaient les risques et partageaient les gains. Ce rapprochement entre intérêts privés et publics demande une explication, car il nous permet de pénétrer, au-delà de la simple question d'organisation fiscale, dans le fonctionnement même de la gestion collective urbaine. À quelques années exceptionnelles près, pendant lesquelles l'initiative privée faisait défaut face au risque jugé trop excessif qui impliquait que l'on se rabattît alors sur la collecte en régie, cette façon de collecter les assises en les affermant était de loin la formule préférée. Elle nous confronte à première vue avec un nouveau paradoxe, car elle persiste bel et bien dans les années pendant lesquelles les élites corporatives participaient au pouvoir, bien que ce fût dans leurs rangs que traditionnellement les opposants les plus farouches à la fiscalité indirecte se retrouvaient. Elle est également à l'origine d'un débat historiographique qui - la privatisation tous azimuts que l'économie et la gestion économique ont connu dans les décennies néo-libérales de la fin du $\mathrm{Xx}^{\mathrm{e}}$ et du début du $\mathrm{XxI}^{\mathrm{e}}$ siècles aidant relève d'un débat d'actualité.

Hans van Werveke, un des derniers élèves directs d'Henri Pirenne, a analysé, dans son étude exemplaire des finances de la ville de Gand au $\mathrm{XIV}^{\mathrm{e}}$ siècle datant des années trente du siècle dernier, les différentes façons de collecter les impôts urbains. Pour lui, la raison pour laquelle les revenus urbains les plus importants (les assises) étaient perçus par des fermiers est à chercher dans la motivation individuelle : les fermiers auraient été plus motivés pour faire rentrer les deniers dans leur caisse, parce qu'ils travaillaient pour leur propre compte et donc pas dans le carcan d'un office public $^{18}$. Un argument pour soutenir cette façon de voir : dans les années où la ville se voyait obligée de recourir à une collecte en régie, le niveau des revenus était toujours plutôt bas. En d'autres termes : l'intérêt privé des fermiers était le carburant garantissant une plus grande productivité et donc des revenus plus importants. D'autres auteurs qui ont étudié la fiscalité des

\footnotetext{
${ }^{17}$ Comme il ressort du mémoire d'Olivier RichaRd, La société, les pouvoirs et le sacré dans les villes $d u$ sud de l'Empire à la fin du Moyen Âge, vol. 3 (essai inédit: Serment et gouvernement dans les villes du Rhin supérieur à la fin du Moyen Age), p. 360-363, Habilitation à Diriger des Recherches soutenue à l'Université de Paris IV, Paris-Sorbonne, le 23 novembre 2015.
} 
villes des anciens Pays-Bas ont bien sûr abordé la question : Raymond van Uytven, qui nous a livré une étude magistrale sur les finances de Louvain, a fortement pesé sur le débat. Pour lui, une plus haute rentabilité due au système de la ferme menait à des revenus plus élevés pour les fermiers, pas forcément pour la ville. Pour lui, et pour J.-P. Peeters, auteur d'une étude sur les finances des petites villes brabançonnes, le choix de donner les assises massivement à ferme était dicté par la nécessité à laquelle la ville devait faire face. En donnant les assises à ferme pour un montant fixe (souvent à payer par mensualités), la ville voyait des revenus fixes arriver dans ses caisses, et en même temps, elle était exempte des dépenses liées à l'organisation d'une collecte en régie ${ }^{19}$. Les recherches pour la ville de Gand pendant la période bourguignonne que j'ai menées et qui ont été complétées pour les dernières décennies $\mathrm{du} \mathrm{xv}^{\mathrm{e}}$ siècle par mon élève Wouter Ryckbosch confirment en général le constat de Van Werveke pour le $\mathrm{XIV}^{\mathrm{e}}$ siècle : la collecte en régie n'était organisée que pendant les années de grandes difficultés (disettes, pénuries, guerres et épidémies) et dans presque tous les cas elle se soldait par une rentabilité nettement inférieure. La question demeure toutefois de savoir si, à côté de ce constat, l'explication mise en avant par Van Werveke et par bien d'autres auteurs est recevable. Est-ce qu'une motivation (et organisation) privée menait automatiquement à une plus grande rentabilité au profit à la fois des fermiers et de la ville? Le débat est difficile, sinon impossible à trancher, tant que nous ne disposons pas des comptabilités privées des personnes en question pour mesurer à la fois l'impact sur les fortunes privées et sur les caisses publiques. Un autre test : l'évaluation de la performance de la collecte en régie directe dans des années «normales» (donc sans un parfum de crise quelconque) fait également défaut. Faux débat donc? Pas

\footnotetext{
${ }^{18}$ H. VAn WerveKe, De Gentsche stadsfinanciën in de middeleeuwen, Bruxelles, 1934, p. 212216, à compléter pour l'époque bourguignonne par M. Boone, Geld en macht. De Gentse stadsfinanciën en de Bourgondische staatsvorming (1384-1453), Gand, 1990 (Verhandelingen der maatschappij voor geschiedenis en oudheidkunde te Gent, XV), passim; et par W. Rycквоsch, Tussen Gavere en Cadzand. De Gentse stadsfinanciën op het einde van de middeleeuwen (1460-1495), Gand, 2007 (Verhandelingen der maatschappij voor geschiedenis en oudheidkunde te Gent, XXXI), passim.
}

${ }^{19} \mathrm{R}$. VAn Uytven, Stadsfinanciën en stadsekonomie te Leuven van de XII tot het einde der $X V I^{e}$ eeuw, Bruxelles, 1961 (Verhandelingen van de Koninklijke Vlaamse Academie voor Wetenschappen, Letteren en Schone Kunsten van België, klasse der Letteren, XXIII, $n^{\circ} 44$ ), p. 125, et J. P. PeEters, De financiën van de kleine en secundaire steden in Brabant van de $12^{\text {de }}$ tot het midden van de $16^{\text {de }}$ eeuw, het voorbeeld van Diest, Nijvel, Tienen, Zoutleeuw, Vilvoorde, Aarschot, Zichem, Geldenaken, Halen, Hannuit, Landen en Gembloers, Bruxelles, 1980, t. 3, p. 650. 
forcément, car l'analyse, non des revenus et des fortunes privées construites éventuellement sur la ferme des impôts urbains indirects, mais bien sur des carrières de fermiers d'assises, peut nous renseigner de façon indirecte sur le milieu social des fermiers et donc sur leurs possibles motivations pour entrer dans le jeu de la ferme des assises. Quels étaient leurs profils professionnels et les autres? Ce sont ces questions que je propose d'aborder à l'aide d'une analyse prosopographique des fermiers d'assises gantois.

\section{Une analyse prosopographique des fermiers gantois}

Des données sont disponibles sans lacune importante pour les années 1400-1455, la période durant laquelle les «trois membres", à savoir bourgeoisie, menus métiers et métiers de la draperie dominaient la vie publique à Gand, à tel point qu'ils se partageaient les mandats politiques, mais également les fonctions de nature publique dans toutes les institutions urbaines, allant de l'administration centrale de la ville jusqu'au niveau des juges subalternes, des contrôleurs de la production économique, voire des receveurs paroissiaux et des institutions caritatives ${ }^{20}$. Avant 1400, les comptes de la ville ne sont malheureusement que partiellement disponibles, tandis qu'après la défaite face au duc de Bourgogne Philippe le Bon en 1453, le système des « trois membres » était en principe aboli et au moins mis hors d'état de fonctionner pour les années à venir. Il est donc légitime de limiter l'analyse en premier lieu à la période des trois membres, pendant laquelle l'influence directe des représentants des corps de métier était à son apogée et importante. Car sur les vingt-six mandats d'échevins à désigner chaque année, vingt étaient entre les mains des représentants ou des métiers liés à la draperie, ou des «menus métiers » (corps de métiers travaillant en premier lieu pour le marché local). La liste nominative des fermiers des impôts indirects nous livre ainsi les noms de 468 individus. Si on fait l'addition de tous les revenus qui passaient entre leurs mains, cela nous donne au total un montant de 32069253 deniers de gros de Flandre, soit en moyenne quelques 68378 deniers par fermier. Comme toujours la moyenne cache une réalité toute autre : $75 \%$ des fermiers ne recouvraient

\footnotetext{
${ }^{20}$ J'ai analysé ce système de partage du pouvoir et des influences dans: M. Boone, «La construction d'un républicanisme urbain. Enjeux de la politique municipale dans les villes flamandes au bas moyen âge ", dans D. Meñot et J.-L. Pinol (éd.), Enjeux et expressions de la politique municipale (XII $-X X^{e}$ siècles). Actes de la $3^{e}$ table ronde internationale du Centre de Recherches Historiques sur la Ville, Paris, L'Harmattan, 1997, p. 41-60.
} 
ensemble que $13 \%$ de tous les revenus, à l'inverse : $6 \%$ des fermiers (33 individus) s'occupaient de la moitié des revenus. Parmi les fermiers une élite restreinte semble donc dominer le jeu. Cette concentration ou tendance oligarchique donne lieu à une courbe de Lorenz, qui en soi ne diffère guère de celle qu'on peut construire sur la base des rares sources de la fiscalité directe, pour un très grand nombre d'élites urbaines médiévales.

Avant de pénétrer dans l'analyse, faisons quelques comparaisons avec d'autres données prosopographiques disponibles. Premier constat, qui ne doit pas forcément surprendre, c'est que malgré le fait que les fermiers manipulaient des sommes considérables et voyaient passer entre leurs mains en moyenne quelque $80 \%$ des revenus de la plus grande ville des anciens Pays-Bas, les "professionnels" du crédit n'arrivaient pas à se tailler une place parmi eux. Si on compare la liste des fermiers avec celle des 182 prêteurs à gages et changeurs connus, dix noms sont identiques (ces dix individus représentent $1,8 \%$ des fermiers et $5,5 \%$ des professionnels du commerce de l'argent), la moitié d'entre eux étant des homonymes $^{21}$. Parmi eux, on ne relève aucun représentant des maisons ou firmes italiennes présentes à Gand. Un premier constat s'impose donc : le monde des professionnels du crédit n'arrivait pas à se mêler à la collecte des deniers publics. Ceci dans l'hypothèse qu'ils auraient voulu faire à Gand ce qui arrivait de temps à temps à Bruges. Nous savons, grâce au travail de Bart Lambert, qu'une entreprise comme celle des Rapondi, financiers originaires de Lucques établis à Bruges, était en mesure et arrivait à avancer une partie des recettes des assises de la ville pour que cette dernière puisse en verser une partie au duc de Bourgogne en payement d'une aide ou d'une autre redevance. Ce jeu financier triangulaire entre ville, prince et financier italien permettait un transfert et une relation financière fluide et profitable pour les trois parties impliquées, et ceci au moins pendant le règne du duc Philippe le Hardi. Quand, sous le principat de son fils et successeur, Jean sans Peur, la situation se détériora en grande partie à cause de l'état de guerre quasi permanent dans lequel l'état bourguignon était impliqué en France -, l'emprise des Rapondi sur les assises brugeoises asphyxiait la ville ${ }^{22}$. À Gand, ville qui en théorie payait au duc presqu'autant que la riche ville commerciale de Bruges selon la répartition fixée par le "Transport de Flandre », une telle mainmise sur

\footnotetext{
${ }^{21}$ J'ai analysé et donné la liste des prêteurs et des changeurs dans M. Boone, "Geldhandel en pandbedrijf in Gent tijdens de Bourgondische periode: politieke, fiscale en sociale aspecten », Belgisch Tijdschrift voor Filologie en Geschiedenis, LXVI, 1988, p. 767-791.
} 
les finances urbaines par un financier étranger ne se produisit pas. En partie parce que, avant la défaite militaire face au duc en 1453, la ville ne payait en fait qu'une partie seulement de ce qu'elle aurait dû payer - et parfois même de ce qu'elle avait consenti à payer -, mais aussi parce que l'élite politique locale avait réussi à mettre la main sur la gestion des finances, et en premier lieu sur la collecte des impôts indirects.

Nous allons donc confronter les listes nominatives des receveurs des assises avec les noms de l'élite politique gantoise, pour mesurer une éventuelle corrélation. Rappelons que dès la grande charte concédée par le comte Gui de Dampierre à la ville de Gand le 8 avril 1297 - en pleine contestation de la gestion financière du patriciat urbain traditionnel -, le cumul d'un mandat d'échevin avec une quelconque participation directe dans l'organisation et l'exploitation des assises était strictement défendu ${ }^{23}$. Mais puisque un mandat d'échevin ne durait qu'une année, et qu'en plus un système de pause entre deux mandats était de mise, le temps et l'opportunité pour les hommes politiques de se lancer dans la gestion des assises étaient réels. Nous n'avons rencontré que trois cas de transgression de l'interdiction du cumul : il s'agissait de trois hommes politiques de tout premier rang. En plus, les trois cas se situaient dans une période avant qu'une mise au point interne, liée à un état d'insurrection en 1432, ne signifie le retour à l'interprétation très stricte des dispositions de $1297^{24}$.

Nous avons retenu comme étant membres de l'élite politique : les échevins (26 par année), les chefs-doyens des membres des menus métiers et de la draperie, au nombre de deux, et les quatre électeurs désignés par la ville pour confirmer formellement les échevins, selon les dispositions de la charte de Senlis. Cette charte contenait un règlement que le roi de France Philippe le Bel avait imposé à la ville de Gand en 1301 et qui est restée la constitution de la ville jusqu'à la Concession caroline de Charles Quint

\footnotetext{
${ }^{22}$ B. LAmBert, The City, the Duke and Their Banker. The Rapondi Family and the Formation of the Burgundian State (1384-1430), Turnhout, Brepols, 2006 (Studies in European Urban History, 7), p. 90-92, 132-137.

${ }^{23}$ A. E. Gheldolf, Les coutumes de la ville de Gand, I, Bruxelles, 1868, p. 499 : « Et le annee ke il seront eschevin, ne porront il estre encherisseurs des maltotes de Gant, ne compaignons ».

${ }^{24}$ Il s'agit de Gelnoot (1424) et Jan van Leyns (1428 et 1430) et de Lievin Papal (1413). Sur les insurrections des années trente : V. FrIS, « De onlusten te Gent in 1432-35 », Bulletin de la société d'histoire et d'archéologie de Gand, VIII, 1900, p. 163 et ss.
} 
en $1540^{25}$. Au total, il s'agit donc de 32 personnes par an, sur une population de 1760 fonctions (pas d'individus, étant donné qu'en moyenne un échevin occupait 3,3 mandats pendant sa carrière politique à cette époque) qu'il faut confronter à nos 468 fermiers/receveurs. De ce groupe, 126 individus $(26,87 \%$, un peu plus qu'un quart) avaient occupé un mandat politique, et totalisaient $37,50 \%$ de tous les revenus. Si on élargit le spectre et que l'on prend en considération, outre les individus qui, sur base de leurs noms et de la chronologie, ont dû avoir une carrière politique et une activité de fermier d'assises en parallèle et qui ont été retenus jusqu'ici, également des membres de leur famille, on peut conclure que 212 fermiers $(45,20 \%$ de la totalité) collectant 55,81\% des revenus de la ville sont à mettre en relation avec l'élite politique gantoise. Allons plus loin et répartissons ces mandataires entre les trois membres de la ville. Constat à première vue étonnant : les représentants des 53 menus métiers (qui travaillent en premier lieu pour le marché local, comme vendeurs de denrées, ou livreurs de services et de travaux, dans le bâtiments, par exemple) dépassent en nombre absolu et en sommes collectées les deux autres membres (bourgeoisie et draperie). Les chiffres sont éloquents : il s'agit, si on s'en tient aux identifications certaines et personnelles, de 29 représentants de la bourgeoisie, de 32 représentants de la draperie et de 65 représentants des menus métiers. Élargis aux possibles liens familiaux, ces chiffres augmentent considérablement, mais gardent la même proportion entre eux : 40 pour la bourgeoisie, 52 pour la draperie, contre 120 pour les menus métiers. Certains liens ne sont pas trop étonnants. Ainsi des bouchers, représentés dans chaque banc échevinal, ont réussi à établir un quasi-monopole sur les assises qui pèsent sur l'utilisation de leur halle marchande, par exemple. Il s'agit d'un corps de métier caractérisé par une hérédité, non pas seulement de fait mais, dans le cas gantois, aussi de droit, situation qui ressemble fort à ce qu'on a pu constater pour d'autres villes médiévales, dont Bruxelles ${ }^{26}$. Si le nombre brut des représentants des membres de la bourgeoisie et de la draperie est presque identique, il en va

\footnotetext{
${ }^{25}$ Une analyse et une édition de cette charte dans M. Boone, «Het "charter van Senlis" (november 1301) voor de stad Gent. Een stedelijke constitutie in het spanningsveld tussen vorst en stad (met uitgave van de tekst) », Handelingen der Maatschappij voor Geschiedenis en Oudheidkunde te Gent, n. r., LVII, 2003, p. 1-45. La charte était considérée comme la constitution de la ville, au point que lorsque le duc Charles le Téméraire a procédé à une humiliation collective de la commune en sa résidence de Bruxelles le 8 janvier 1469, la charte a été cancellée lors d'une session publique de la cour de Bourgogne. Pour l'indiciaire Georges Chastellain, cette séance théâtrale comptait parmi les douze magnificences du duc en question : W. Paravicinı, " Die zwölf "magnificences" Karls des Kühnen », Vorträge und Forschungen, LI, 2001, p. 323-327.
} 
différemment pour les sommes dont ils ont été les dépositaires. La moyenne des revenus réalisés par des hommes politiques au nom de la draperie était la moitié de ce qu'ont pu manipuler les représentants de la bourgeoisie (52 314 deniers contre 141807 deniers). Autre différence, chez les représentants des menus métiers, le nombre de fermiers qui n'occupent qu'un seul mandat politique est nettement supérieur aux deux autres membres, sans que cela signifie qu'ils aient pris à ferme des assises pour des montants dérisoires. Souvent celui qui n'était qu'une seule fois échevin au nom d'un des 53 menus métiers prenait à ferme une assise importante. Le nombre plus élevé de possibles candidats de corps de métier à représenter au sein de ce membre et une solvabilité plus grande et plus diversifiée, peuvent expliquer cet état de choses.

Si nous nous attardons sur le groupe restreint déjà évoqué des 32 fermiers qui se situent au sommet, du fait qu'ils sont responsables pour $50 \%$ de la totalité des revenus ${ }^{27}$, nous constatons qu'ils reflètent l'image globale : plus de mandats politiques par individu pour ceux appartenant à la bourgeoisie et à la draperie, un nombre absolu plus important pour les représentants des menus métiers. Au sein de chaque groupe, des liens familiaux très forts font surface. Des familles, telles les Van Leyns dans la draperie, les De Grutere pour la bourgeoisie et les Papals pour les menus métiers, semblent s'être durablement installés dans les activités de fermiers d'assises. Toutefois, sur les 32 fermiers, huit n'ont visiblement aucun lien direct avec un des trois membres de la ville. Ce qui devrait nous mettre en garde, élite fiscale et élite politique ne se recoupent pas intégralement : un succès comme fermier était possible sans soutien ou lien politique.

Troisième banque de données avec laquelle on peut recouper la liste des fermiers des assises: celle concernant les entrepreneurs qui ont effectué des livraisons de vin, de draps, de bois et/ou de tourbe; de parchemin, papier, encre, etc. pour l'administration; de matériaux de construction, etc., à la ville ${ }^{28}$. Après une comparaison systématique, il apparaît que seuls 86 des 468 fermiers de notre "population» (soit

${ }^{26}$ Voir (avec des références à la littérature plus datée sur Gand) : C. Deligne, C. BIllen, D. Kusman, «Les bouchers bruxellois au bas Moyen Âge. Profils d'entrepreneurs », dans S. Jaumain et K. Bertrams (dir.), Patrons, gens d'affaires et banquiers. Hommages à Ginette Kurgan-Van Hentenryck, Bruxelles, Le Livre Timperman, 2004, p. 69-92.

${ }^{27}$ Avec comme limite 280000 deniers par individu, ce qui équivaut à un cinquième des revenus annuels dans leur totalité ou la moitié en moyenne des dépenses administratives annuelles de la ville. 
$18,38 \%$ ) ont été enregistrés comme fournisseurs. Le nombre de personnes ayant été fermier et fournisseur la même année est nettement inférieur : seulement 19 des 86. Ce qui indique qu'il n'existait pas une situation de collusion systématique, au contraire : si on prend en considération la chronologie, on ne peut que constater que dans beaucoup de cas une carrière de fournisseur est interrompue au moment où le commerçant et/ou entrepreneur prend à ferme une des assises de la ville. Aucun règlement interne («voorgebod» comme on les appelait à Gand) n'est connu qui défende expressément un tel mélange d'activités lucratives, mais on pourrait supposer, étant donné l'interdiction globalement bien respectée de ne pas agir comme fermier quand on était élu échevin, que cette interdiction avait trouvé une application plus large, menant à une certaine retenue parmi les fournisseurs ${ }^{29}$. Dans le contexte d'une ville où les influences politiques avaient gardé un lien direct avec les activités économiques par la présence importante des représentants des corps de métier à tous les niveaux de la gestion et du gouvernement, une possible concussion généralisée par la manipulation de la fiscalité indirecte à son profit, faussant ainsi une concurrence "normale», aurait déstabilisé les équilibres subtils sur lesquels reposait le système politique gantois. Une préoccupation pour les édiles gantois, car si la ville se trouvait souvent à l'avant-garde de l'opposition à la politique princière, cette grande tradition révolutionnaire était soutenue par une "petite» tradition de révoltes internes, alimentée par une sensibilité politique et fiscale aiguë ${ }^{30}$.

\footnotetext{
${ }^{28}$ Des considérations autour de la notion de livraisons, et concernant une éventuelle concussion: M. Boone, "Gestion urbaine, gestion d'entreprises : l'élite urbaine entre pouvoir d'État, solidarité communale et intérêts privés dans les Pays-Bas méridionaux à l'époque bourguignonne ( $\mathrm{xIV}^{\mathrm{e}}-\mathrm{Xv}^{\mathrm{e}} \mathrm{s}$.) », dans S. CAVACIOCCHI (éd.), L'impresa. Industria, commercio, banca secc. XIII-XVIII (Prato, istituto internazionale di storia economica F. Datini, serie II, Atti della XXIIa settimana di studi 30 aprile-4 maggio 1990), Firenze, 1991, p. 853-854.
}

${ }^{29}$ Sur les règlements d'ordre policier émanant des échevins en tant que législateurs : M. BOONE, "Législation communale et ingérence princière : la "restriction" de Charles le Téméraire pour la ville de Gand (13 juillet 1468) », dans J.-M. Cauchies et E. Bousmar (éd.), "Faire bans, edictz et statuz" : légiférer dans la ville médiévale. Sources, objets et acteurs de l'activité législative communale en Occident, ca. 1200-1500. Actes du colloque international tenu à Bruxelles les 17-20 novembre 1999, Bruxelles, 2001 (Publications des Facultés universitaires Saint-Louis, vol. 87), p. 139-151.

${ }^{30}$ Voir M. Boone, M. Prak, «Rulers, Patricians and Burghers: the Great and the Little Traditions of Urban Revolt in the Low Countries ", dans K. Davids et J. LuCASSEN (éd.), A Miracle Mirrored. The Dutch Republic in European Perspective, Cambridge, Cambridge University Press, 1995, p. 99-134. 
Un dernier aspect que l'approche prosopographique nous a permis d'aborder de façon plus approfondie, consiste dans l'analyse - limitée dans le temps il est vrai, tant la situation heuristique est moins favorable sur ce point précis -, de la façon dont les assises furent accordées à un fermier précis, processus qui tournait essentiellement autour de la possibilité du fermier en question de donner des gages et des garanties suffisants à la ville. Pour les années 1404-1436, un registre contenant à la fois les règlements internes et les listes des personnes qui se portent garantes pour les fermiers des assises a été conservé ${ }^{31}$. L'analyse n'en a pas encore été faite de façon aussi approfondie que pour les autres dossiers, le nombre de liens à scruter étant plutôt démotivant ${ }^{32}$. Quelques sondages confirment toutefois l'impression générale que si les fermiers appartiennent souvent à des familles bien en vue et bien implantées parmi les élites bourgeoises et corporatives de la ville, cet engagement familial (famille au sens large) s'étend à un secteur à première vue éloigné de la solidarité familiale : l'affermage des impôts indirects. Le fait que des fermiers affichent une même préférence politique (en faveur de la politique centralisatrice du duc de Bourgogne par exemple) peut également, comme ce fut le cas en 1410, souder un groupe d'individus qui s'associent temporairement pour prendre à ferme les assises les plus importantes de la ville qui fut souvent le pilier d'une opposition particulariste vis-à-vis du duc. Autre constat qui s'impose: à plusieurs reprises, les mêmes personnes peuvent être identifiées comme garantes des fermiers. Parfois on se porte garant pour le même fermier, même si celui-ci prend à ferme plusieurs assises dans la même année. Les garants ne deviennent pas (ou alors très rarement) fermiers eux-mêmes, et parmi eux on ne trouve pas de professionnels de la gestion financière ni de représentants des maisons italiennes de changeurs ou de banquiers. La recherche ultérieure devrait s'intéresser davantage à ce groupe d'acteurs.

\footnotetext{
${ }^{31}$ Il s'agit du registre : Archives de la ville de Gand, série 108, ${ }^{\circ} 2$, passim. Une analyse dans : M. Boone, « Triomferend privé-initiatief versus haperend overheidsoptreden ?», art. cit., p. 132-133.

${ }^{32}$ Un exemple : pour l'assise sur la bière d'origine étrangère en 1404. Rien que pour cet impôt, sept associés agissant comme fermiers et sept garants sont mentionnés. Un tel nombre se rencontre pour les six assises les plus importantes. Comme nous disposons des données pour 27 années, (entre 1404 et 1436), cela implique que rien que pour les assises qui touchent le vin ( 4 assises) et la bière $(2$ assises $), 2268$ relations $(27 \times 6 \times 14)$ deviennent un sujet de recherche.
} 


\section{En guise de conclusion}

Des analyses prosopographiques, aussi bien dans le cas d'une grande ville que pour des villes de taille moyenne ou carrément petites, ont démontré à quel point les représentants des corps de métiers se sont installés dans le système d'affermage des assises, et ont donc pu en profiter pleinement ${ }^{33}$. S'engager dans la collecte des impôts s'est avéré être à long terme un investissement qui aidait certaines familles à augmenter sensiblement leur capital social à l'intérieur d'une ville. C'est ce qui ressort d'études pour les villes flamandes et brabançonnes, mais également pour les modestes capitales provinciales que furent Namur ou Luxembourg ${ }^{34}$. On imagine donc facilement que les élites corporatives n'ont pas trop ressenti le besoin de pousser à fond la critique du système fiscal qui revenait régulièrement à la surface parmi leurs membres. D'autant plus que la fiscalité indirecte restait, compte tenu des possibilités administratives de l'époque, l'unique moyen pour répondre aux besoins. Cela reflète bien sûr un choix politique : les élites urbaines des anciens Pays-Bas n'ont pas investi dans le développement poussé d'une administration qui aurait permis l'établissement d'une fiscalité type « estime », voire « catasto». Il faut d'ailleurs bien constater que même dans les villes italiennes, le catasto n'a jamais remplacé le poids écrasant de la fiscalité indirecte ${ }^{35}$. La levée d'impôts indirects, avec l'affermage comme formule d'exploitation par excellence, offrait aux élites au pouvoir, qu'elles aient été de nature corporative ou patricienne, trop de possibilités d'investissement, de retour sur investissement et de construction d'une cohésion interne, pour être abandonnée.

\footnotetext{
${ }^{33}$ Voir pour deux petites villes du Quartier gantois : P. STABEL, « Van schepenen en ontvangers. Politieke elite en stadsfinanciën in Axel en Hulst », Tijdschrift voor sociale geschiedenis, 18,1992 , p. 1-21.

${ }^{34}$ C. Dickstein-Bernard, La gestion financière d'une capitale à ses débuts : Bruxelles, 13341467, Bruxelles, 1977, passim; M. TreiYer-LiÉnARD, « La gestion des finances de la ville de Namur (1362-1477) », dans M. Boone et W. PrevenIER (éd.), Finances publiques et finances privées au bas moyen âge. Actes du colloque tenu à Gand les 5 et 6 mai 1995, LeuvenApeldoorn, Garant, 1996, p. 131-161 (l'auteure a présenté quelques années plus tard une thèse sur les finances de Namur restée inédite jusqu'ici, Université de Louvain-la-Neuve) ; M. Pauly, «Les familles scabinales et la gestion des finances à Luxembourg au $\mathrm{xv}^{\mathrm{e}}$ siècle ", dans Finances publiques et finances privées, op. cit., p. 163-189.
}

${ }^{35}$ Je renvoie à la mise en garde de J.-C. Maire-VIGueur, «Les rapports ville-campagne dans l'Italie communale : pour une révision des problèmes ", dans N. Bulst, J.-Рн. Genet (éd.), La ville, la bourgeoisie, et la genèse de l'État moderne (XII ${ }^{e}-X V I I I^{e}$ siècles), Paris, CNRS, 1988, p. 24-28. 
